Revisão

\title{
Tendências da produção científica sobre o tráfico de espécies
}

\author{
Letícia Pereira dos Santos ${ }^{1 *}$, Danielle Rodrigues de Araujo ${ }^{1}$ \\ ${ }_{1}^{1}$ Programa de Pós-Graduação em Ecologia e Evolução, Universidade Federal de Goiás, GO, Brasil. *Autor para correspondência: santospear@gmail.com \\ ${ }^{2}$ Programa de Pós-Graduação em Recursos Naturais do Cerrado, Universidade Estadual de Goiás, GO, Brasil.
}

\section{N F O A R T I G O}

Histórico do artigo

Recebido: 08 agosto 2015

Aceito: 07 setembro 2015

\section{Palavras chaves:}

Espécies ameaçadas

Lista vermelha

Mamíferos

Vieses

\begin{abstract}
A B S T R A C T
The aim of this study was to evaluate trends in global research about trafficking of species through a scientometric analysis. Was carried a search for scientific articles in Web of Science database on the subject illegal trade of species published between the years 1991 to 2012 . We found 254 publications, of which no trend in the number of articles was found over the years. However, publications on ecological, conservation and genetic issues presented temporal increase. There is still a concern of researchers to check the ecological impacts and the importance of conserving biodiversity using molecular tools that allow the identification of species traded illegally. Furthermore, the relationship between the species and the final interest, for which it is marketed, can provide important data to contribute to the supervision of illegal trade of species.
\end{abstract}

\section{Introdução}

O tráfico de espécies silvestres é uma prática antiga e que tem promovido uma grande redução na biodiversidade global (Redford, 1992; García \& Suárez, 2000). Essa prática ilegal agride de maneira severa o meio ambiente, causando perda faunística, extinção local de espécies e até mesmo desestruturação de ecossistemas (WWF, 1995; IBGE, 2004). Em consequência a essas reduções, impactos significativos podem ser gerados sobre a estrutura de comunidades, provocando efeitos irreversíveis e diminuição da função ecológica do ambiente (Ribeiro \& Silva, 2002).

As várias espécies de animais, vertebrados e invertebrados, traficadas são comercializadas tanto em âmbito nacional quanto internacional, utilizadas para diversas finalidades (RENCTAS, 2007). Em geral, a comercialização ilegal de espécies silvestres é destinada principalmente a zoológicos, pets shops, colecionadores, indústrias e também para a pesquisa científica (Prado \& Malheiros, 2012). Algumas por exemplo são empregadas a componentes fundamentais de potencial farmacêutico, de confecção de artesanatos, ou ainda são destinadas como animais de estimação (Hathaway, 2004; Bastos et al., 2008).

Apesar dos prejuízos ecológicos causados pelo tráfico de espécies, pouco se sabe sobre as características que regem essa prática (RENCTAS, 2001). Além disso, as medidas tomadas para mitigar esse comércio ainda são falhas e, em algumas regiões do mundo, inexistentes (Saab, 2006; RENCTAS, 2007). Isso se deve principalmente ao seu carácter ilegal, o que torna difícil determinar com precisão dados quantitativos e categóricos que poderiam auxiliar na mitigação ou combate a essa prática (RENCTAS, 2001; BarberMeyer, 2010).

A cienciometria consiste em uma análise quantitativa de investigação da literatura que avalia o estado da arte no campo científico (Spinak, 1998). Estudos cienciométricos têm sido realizados em várias áreas visando determinar as tendências e vieses que as norteiam (Macias-Chapula, 1998; Lima-Ribeiro et al., 2007; Carneiro et al., 2008; Brito et al., 2009; Barbosa et al., 2012; Nabout et al., 2012). Esse tipo de avaliação é realizado por meio de indicadores cienciométricos que embasam as análises e proporcionam a obtenção de resultados (Spinak, 1998).

A cienciometria tem como principal tema de interesse o crescimento quantitativo do número de estudos sobre um determinado tema em escala temporal, levando em consideração fatores sociais, econômicos e culturais (Spinak, 1998). Nesse contexto, julga-se relevante a realização de um estudo que busque avaliar quantitativamente a pesquisa científica à cerca do tráfico e comércio ilegal de espécies.

Por tais motivos este estudo tem como principal objetivo verificar a tendência temporal do tráfico de espécies, com foco em animais silvestres. Especificamente verificou-se a tendência temporal no número de artigos publicados; a nacionalidade do primeiro autor; o número de artigos publicados para cada grupo taxonômico vítima de tráfico; a existência de relação entre os grupos e os fins pelos quais o tráfico de espécies ocorre; a relação do número de artigos, os 
níveis de ameaça das espécies e; as tendências das áreas de pesquisa ao longo dos anos.

\section{Material e Métodos}

0 estudo foi conduzido por meio de uma busca realizada na base de dados ISI Web of Science (http://apps.webofknowledge.com), na qual foram considerados trabalhos que abrangessem o tema tráfico e comércio ilegal de espécies. Foram utilizados todos os tipos de publicações (artigos, livro, revisões, cartas, dentre outros) indexadas a esta base e publicadas entre os anos de 1991 a 2012.

Para a realização da busca utilizou-se as seguintes palavras: "illegal* trade*" OR "trafficking* species" OR "trad* ilegal*" OR "ilegal trad*" OR "traffick species" OR "biopirac*" OR "ilegal wildlife trade" OR "animal* poach*" OR "illegal animal* hunt*" OR "poach*", inseridas no campo de busca por Title. As palavras escolhidas foram usadas com o intuito de refinar a pesquisa apenas com publicações que tem como temas principais o tráfico e contrabando de espécies.

As análises dos resumos das publicações foram feitas de acordo com (i) o ano de publicação; (ii) principais temas de estudo, definido com base nos objetivos dos trabalhos; (iii) grupo biológico (plantas, mamíferos, aves, répteis, invertebrados, peixes); (vi) interesse final para os quais as espécies são comercializadas, e; (v) o nível de ameaça das espécies. Para obter o nível de ameaça das espécies foram utilizados dados contidos nos resumos e na Lista Vermelha disponibilizada pela União Internacional para Conservação da Natureza (IUCN, 2013).

A tendência temporal no número de artigos entre os anos de 1991 a 2012 foi verificada por meio de uma correlação de Spearman. Com o intuito de remover a tendência natural do número de publicações, foi dividido o número de artigos publicados sobre o tráfico de espécies pelo número total de artigos publicados na base de dados ISI em todos os anos e, em seguida, esse valor foi multiplicado por 100 , gerando um valor percentual.

Da mesma forma, para analisar as tendências temporais dos principais temas de estudos para o mesmo período foi utilizada uma correlação de Spearman. Além disso, a fim de verificar se existe diferença na média do número de artigos, quanto à abordagem de espécies em alguma categoria de ameaça em extinção (ameaçada, vulnerável, criticamente ameaçada), com os estudos que não abordam espécies ameaçadas (categoria de menor preocupação de acordo com a IUCN), foi usado um teste não paramétrico de Mann-Whitney (U). Os testes estatísticos foram realizados com nível de significância $\alpha$ (alfa) de 5\% e no programa livre R Core Team (2011).

Para verificar se existe relação do grupo taxonômico com o interesse final de tráfico, foi realizado uma Análise de Correspondência. 0 resultado da análise pode ser observado em um gráfico bidimensional, em que a variação dos dados é obtida em porcentagem para os dois principais eixos (Greenacre \& Hastie, 1987). Dessa forma é possível observar as distribuições das amostras (grupo taxonômico) com suas variáveis (interesse final) observadas mais frequentes.

\section{Resultados}

Entre 1991 e 2012 foram encontradas, no total, 254 publicações sobre tráfico de espécies, publicadas em 147 revistas indexadas à base de dados do ISI Web of Science. Publicação do tipo artigo foi a mais frequente $(\mathrm{N}=125$; 49,21\%), seguida de Book Review ( $\mathrm{N}=54 ; 21,25 \%)$ e News Item $(\mathrm{N}=28 ; 11,02 \%)$.

Não houve relação significativa do número de artigos ao longo dos anos $(\mathrm{r}=-0.19 ; \mathrm{P}=0.37 ; \mathrm{N}=254)$. Porém foi possível notar a presença de três picos de crescimento das publicações nos anos de 1992, 1999 e 2002 (Figura 1).

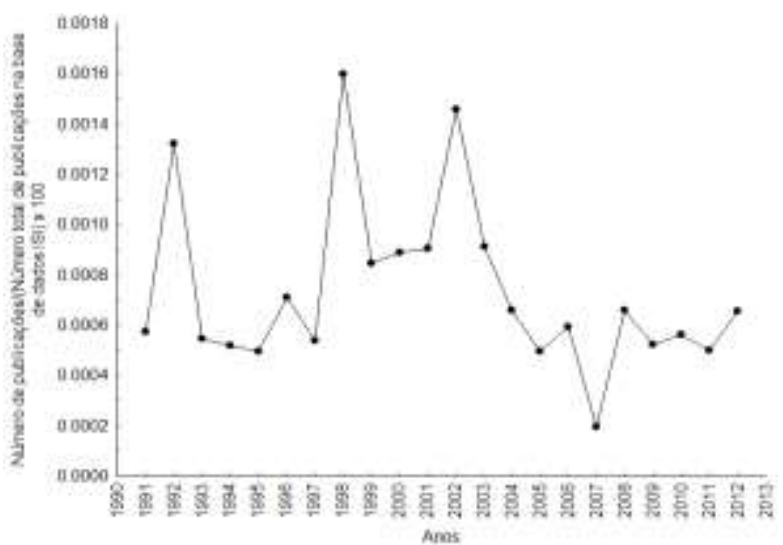

Figura 1. Tendência temporal do número de publicações indexadas na base de dados ISI Web of Science sobre o tema tráfico de espécies entre os anos de 1990 a 2012.

Os Estados Unidos é o país que possui o maior número de autores $(\mathrm{N}=79 ; 63,2 \%)$ publicando sobre o tema tráfico de espécies (Figura 2), seguido da Inglaterra $(\mathrm{N}=20$; $16 \%)$. 0 Brasil ocupa a 7 a posição $\operatorname{com} 3,2 \%(\mathrm{~N}=4)$ das publicações.

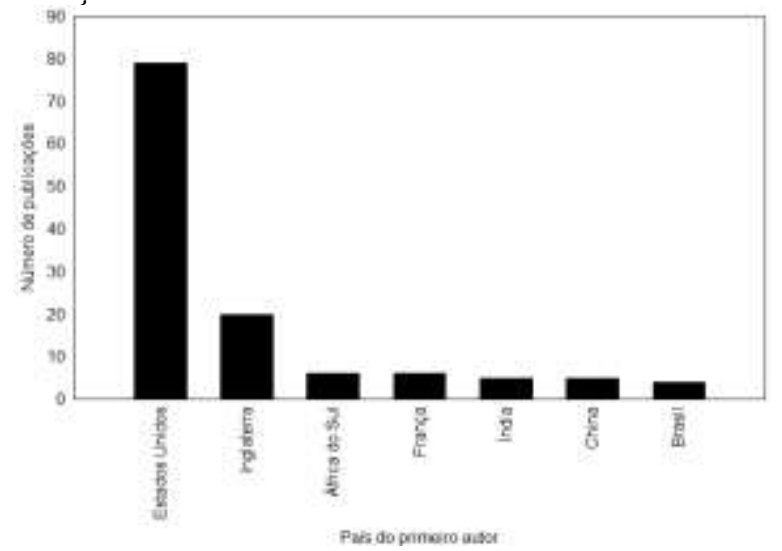

Figura 2. Número de publicações na base de dados do ISI Web of Science entre os anos de 1991 a 2012 sobre o tema tráfico de espécies em relação à nacionalidade do primeiro autor.

O grupo biológico mais frequente nos temas de estudo foram os mamíferos, com 80 registros (45,9\%). Em seguida está o grupo das aves como o segundo mais estudado em tráfico de espécies $(\mathrm{N}=19 ; 10,73 \%)$. Peixes e a herpetofauna concentraram em conjunto $11,30 \%(\mathrm{~N}=20)$ das publicações. Os invertebrados, que incluem moluscos, corais e insetos, possuem o menor número de registros, com apenas quatro publicações referentes ao tema do estudo $(2,25 \%)$ (Figura 3).

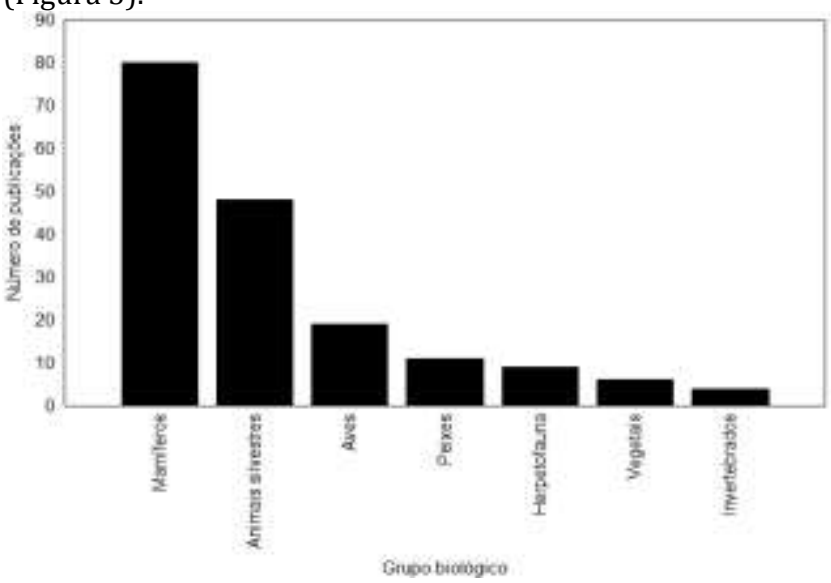

Figura 3. Número de publicações por grupo biológico sobre o tema tráfico de espécies na base de dados ISI Web of Science entre os anos de 1991 a 2012. 
Além disso, observa-se que há uma relação do táxon com o interesse final que o leva ao comércio ilegal. 0 primeiro eixo da análise de correspondência explicou 45,06\% da variação dos dados e o segundo eixo $34,25 \%$ (Figura 4). Em geral, os trabalhos realizados com mamíferos estão mais relacionados com artesanato, peixes com alimentação, herpetofauna agrega interesse em pesquisa e fins alimentícios, aves são comercializadas principalmente para estimação, e plantas têm relação com outros interesses, como por exemplo, para fins madeireiros.

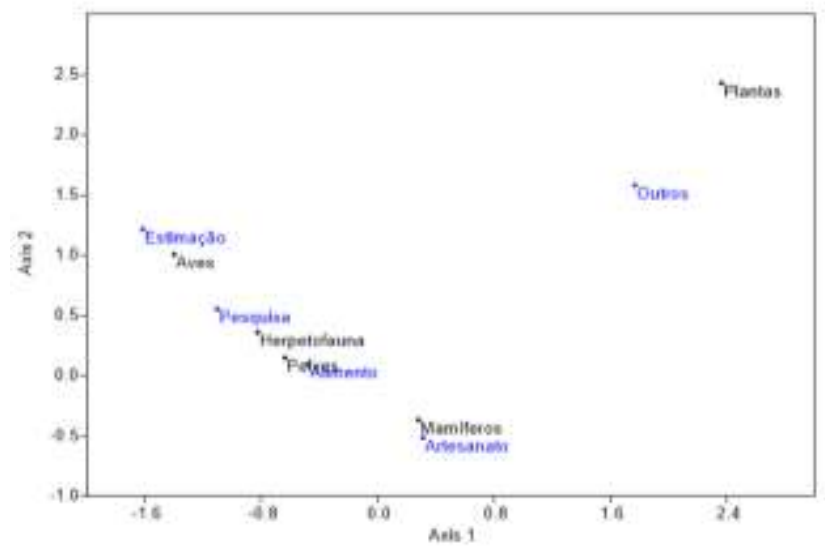

Figura 4. Análise de correspondência demonstrando a relação entre os grupos taxonômicos acometidos pelo tráfico e categorias de interesse final, de acordo com publicações na base de dados ISI Web of Science entre os anos de 1991 a 2012.

Em média existe uma diferença no número de artigos quanto à abordagem do nível de ameaça das espécies com os trabalhos que não abordam nenhuma categoria de ameaça (U= $145 ; \mathrm{p}=0.02)$. A maioria dos autores $(28,74 \% ; \mathrm{N}=73$ ) concentra suas pesquisas em espécies sem riscos e não ameaçadas de extinção. Enquanto os demais autores (14,56\%; $\mathrm{N}=37$ ) realizam seus estudos com espécies que estão ameaçadas em algum nível segundo a IUCN.

Os principais temas abordados nos trabalhos (Tabela 1) correspondem a $78,74 \%(\mathrm{~N}=200)$ das publicações. As publicações que contém temas sobre ecologia de espécies, aspectos da importância da conservação de espécies vítimas do tráfico e assuntos do meio socioeconômico apresentaram picos de maior crescimento no ano de 2002. Apenas os assuntos ecológicos, conservacionistas e genéticos apresentaram um aumento temporal no número de artigos. Os temas socioeconômicos, políticos e sobre biopirataria não apresentaram relações significativas ao longo dos anos (Tabela 1).

Tabela 1. Número de publicações e tendências dos estudos abordados sobre tráfico de espécies. Valor da correlação de Spearman e nível de significância para cada variável analisada temporalmente.

\begin{tabular}{lccc}
\hline Tema de estudo & $\begin{array}{c}\mathbf{N}^{\circ} \text { de } \\
\text { publicações }\end{array}$ & $\begin{array}{c}\text { Correlação } \\
\text { de } \\
\text { Spearman }\end{array}$ & $\begin{array}{c}\text { Valor de } \\
\mathbf{P}\end{array}$ \\
\hline Ecológico & 42 & 0.45 & 0.03 \\
Político & 42 & 0.24 & 0.28 \\
Socioeconômico & 32 & 0.09 & 0.66 \\
Conservação & 31 & 0.48 & 0.02 \\
Biopirataria & 29 & 0.34 & 0.11 \\
Genético & 22 & 0.74 & $<0.006$ \\
\hline
\end{tabular}

Alguns temas foram perdidos e não analisados, pela falta de informação suficiente para classificá-los. Este problema deve-se ao fato de alguns tipos de publicações não oferecerem o resumo das pesquisas.

\section{Discussão}

4.1. Produção científica
A relevância em uma área de pesquisa depende do número de pesquisadores que aborda determinado tema (Peters, 1991). No entanto existe uma grande dificuldade em se obter informações em níveis globais, regionais ou locais sobre o comércio ilegal de vida selvagem (Hansen et al., 2012; Barber-Meyer, 2010). Tal fato pode explicar a estabilidade no número de publicações ao longo dos anos. Desta forma, podemos considerar que o interesse da comunidade científica sobre determinado tema está relacionado à forma de obtenção de dados, o qual, para tráfico de espécies, muitas vezes não existem ou são de acesso restrito a gestores ambientais.

Além da dificuldade e falta de precisão das pesquisas decorrente da prática clandestina de comércio de espécies, a política de fiscalização restringe os resultados para a pesquisa científica de tráfico de espécies. Barber-Meyer (2010), em sua pesquisa sobre comércio ilegal de espécies observou uma queda na taxa de ocorrência de tráfico de espécies em $20 \%$ dos municípios nos Estados Unidos (EUA). Tal observação resulta da abordagem de políticas conservacionistas que revigora a fiscalização do comércio clandestino no país (Barber-Meyer, 2010).

De acordo com May (1998) os Estados Unidos e os países da União Europeia estão entre as nações que mais investem em infraestrutura e pesquisa científica. Outros estudos cienciométricos, também têm encontrado a nacionalidade norte-americana de primeira autoria como a mais frequente nas publicações científicas, em diferentes áreas de pesquisas (Carneiroet al., 2008). De fato, países industrializados de grande poder econômico fornecem mais subsídios tecnológicos e educativos que permitem o crescimento na pesquisa científica (Glänzel et al., 2006).

Além disso, no estudo de monitoramento do comércio ilegal de vida selvagem de Hansen et al. (2012), os Estados Unidos ocupam a segunda posição de país com maior registro de comércio ilegal de espécies (15,3\%), ficando atrás apenas da Índia $(15,6 \%)$. Com maior investimento em pesquisas e maior ocorrência de comércio ilegal de vida, de fato esperava-se que os Estados Unidos ocupassem o primeiro lugar no ranking mundial de autores que publicam sobre o tema.

No Brasil, a pesquisa científica é independente do setor de produção nacional, e possui pouco investimento, o que desfavorece a produção nas ciências e, consequentemente, faz com que um menor número de pesquisadores ingresse na carreira científica (Schwartzman, 2003). Contudo, apesar da carência nos sistemas de tecnologia e educação, o Brasil possui uma taxa de crescimento de $8 \%$ das pesquisas científicas mundiais, além de ocupar a primeira posição no número de publicações anuais na América Latina (Glänzel et al., 2006).

\subsection{Tendências das pesquisas}

A maior concentração das pesquisas sobre tráfico de animais na classe Mammalia, provavelmente, refere-se ao interesse final das espécies vítimas do tráfico (TRAFFIC, 2008), que acabam por oferecer mais dados para as pesquisas científicas. Dos estudos com espécies de mamíferos foi observado que, tanto o animal vivo, quanto partes de seu organismo podem ser comercializados. Pesquisas com espécies da ordem Artiodactyla (ungulados), por exemplo, mencionam interesse para consumo humano. Além disso, outras partes, como peles e couros são úteis na fabricação de ornamentos e utensílios em geral. Além disso, muitos mamíferos também têm sido capturados para fins medicinais (Hansen et al., 2012).

Dentro do grupo dos mamíferos a espécie de elefante africano Loxodonta africana obteve o maior número de pesquisa, compreendendo 33,33\% $(\mathrm{N}=11)$ dos estudos. Além de Loxodonta africana, outras espécies de elefantes e rinoceronte concentraram $15,35 \%$ das pesquisas totais publicadas. Hansen et al. (2012) encontraram resultados 
similares para as mesmas espécies vítimas da caça ilegal. Espécies de elefantes obtiveram maior número de registros de comércio interceptado seguido de registros ilegais de caça de rinocerontes. 0 principal interesse observado nas pesquisas pelo comércio e caça ilegal destas espécies referem-se ao uso ou fabricação de ornamentos pela venda do marfim de elefantes e chifres de rinocerontes.

Sabe-se que o chifre de rinocerontes possui alto valor de mercado, o que contribui para o aumento da caça furtiva e o comércio ilegal (Ferreira \& Okita-Ouma, 2012). Com relação às espécies de elefantes, Maingi et al. (2012) relacionam a caça furtiva com comércio de marfim e consumo da carne no Quênia. Além disso, estes autores relatam que a facilidade ao ponto de acesso a estes animais na natureza contribui para o aumento da caça.

Há uma grande abordagem nas pesquisas sobre venda ilegal de partes de animais voltado para o comércio de ornamentos. Entretanto existe um viés quanto a esse resultado. Espécies de elefantes e rinocerontes foram as mais frequentes nas pesquisas sobre tráfico de espécies, cujo interesse final observado é o comércio ilegal de marfim, no uso de ornamentos e artesanatos em geral. Assim, interesse de mamíferos voltados para o consumo de carne, por exemplo, pode estar subestimado devido ao grande número de publicações que relaciona elefantes e rinocerontes com o interesse, principalmente, para o artesanato.

Muitos trabalhos não se referiram em específico à espécie ou ao grupo taxonômico em estudo. Nestas pesquisas os autores apenas mencionavam nos resumos das pesquisas as palavras "vida selvagem" ou "animais silvestres". 0 objeto de estudo desses autores não se referia ao comércio ou caça ilegal de um grupo específico, mas sim apresentavam uma abordagem mais ampla dessa prática ilegal. Barbanera et al. (2012), por exemplo, aborda comércio ilegal de vida selvagem, cujo tema central refere-se a técnicas moleculares de investigação de espécies vítimas do tráfico.

Aves foi o segundo grupo taxonômico com maior registro de comércio ilegal nas pesquisas, cujo principal interesse voltado a animais de estimação. De acordo com Pires e Clarke (2011) espécies deste grupo possuem alta abundância na natureza, são acessíveis para caça e coleta, e agrada ao público que as obtém como animais de estimação. Tais fatores contribuem para o aumento do comércio ilegal desses animais, principalmente de espécies raras ou em risco de extinção, das quais conferem aparência e cantos atraentes (Destro et al., 2012; Ferreira \& Glock, 2004).

Com menor número de publicação alguns autores relatam sobre o tráfico de espécies de peixes e herpetofauna, deste último, principalmente de tartarugas. Obtenção como animais de estimação, pesquisa e principalmente alimentação (incluindo também os peixes) foram os interesses de comercialização de tartarugas observados na literatura. Gong et al. (2006) também encontraram resultado similar, descrevendo os mesmos interesses para as espécies de tartarugas.

Com base na categoria de ameaça das espécies segundo a lista vermelha da IUCN foi observado que a grande maioria dos autores não aborda em suas pesquisas o nível de ameaça das espécies, ou relatam sobre tráfico cujas espécies possuem menor preocupação quanto ao nível de ameaça. Em contrapartida, de acordo com a RENCTAS (2001) as espécies que possuem maior nível de ameaça são as mais comercializadas e que possuem maior valor no mercado clandestino. Entretanto, não necessariamente as espécies observadas nos estudos apresentam status de menor preocupação. 0 comércio ilegal de espécies também possui uma forte relação com o nível de ameaça das espécies, que pode levar à extinção (Chaudhary, 2004). Muitos estudos, no entanto, citaram o nome comum da espécie que, com esta condição, não foi possível obter as categorias de ameaça pela lista vermelha. Com isso acabam por se classificar em conjunto com os estudos que mencionam espécies que não possuem risco de extinção ou nenhum grau de ameaça.

A tendência no aumento temporal nos principais temas abordados na literatura sobre tráfico de espécies foi observada para pesquisas com assuntos que abordam aspectos da prática de conservação de espécies, ecológicos e da biologia molecular. Para o ano de 2002 foi observado um pico de aumento nas pesquisas de temas sobre ecologia e política. Este crescimento se deve por conta que neste período $45 \%$ das publicações encontradas foram do tipo Book Review de um mesmo estudo que abordam estas mesmas questões.

Os temas sobre conservação e ecologia exploram principalmente estratégias para proteção de espécies vítimas do tráfico, índices de mortalidades, características comportamentais, além do seu estado de conservação frente a essa prática ilegal. As tendências de crescimento das pesquisas ecológicas e conservacionistas referem-se quanto à sua importância em compreender, os impactos gerados sobre as espécies e informar o nível de exploração das espécies e a importância ecológica em se conservar a fauna em seu hábitat natural (Kuhl et al., 2009).

Os estudos de biologia molecular fornecem e informam importantes ferramentas na identificação por meio da genética forense de animais vítimas do tráfico, o que pode conferir meios eficazes de monitorar e fiscalizar o comércio ilegal de animais silvestres (Barbanera et al., 2012).

A comunidade científica abordou com frequência os temas políticos e socioeconômicos de tráfico de espécies. Entretanto, não se observou nenhuma tendência significativa de crescimento e ou diminuição no decorrer dos anos das publicações. As pesquisas na área de humanas abordam principalmente os perfis socioeconômicos de fornecedores e comerciantes de espécies. Além disso, aspectos políticos que regem as leis de crime ambiental, como o comércio ilegal, a caça furtiva e propriedade intelectual (biopirataria) foram observados nas pesquisas. Kuhl et al. (2009) concluíram em seu estudo, que o comércio ilegal de espécies silvestres é praticado principalmente por moradores de zona rural que possuem baixa renda econômica e que necessitam do lucro obtido pela venda de espécies.

\section{Conclusões}

Análises cienciométricas permitem verificar as principais tendências das publicações sobre determinado tema. Os resultados aqui apresentados com base na literatura demonstram que as pesquisas não têm oferecido uma tendência temporal no número de publicações sobre tráfico de espécies. Em contrapartida, há uma preocupação dos pesquisadores em verificar os impactos ecológicos e a importância de conservação e proteção das espécies, por meio de fiscalização e ferramentas moleculares que permitem a identificação de espécies ameaçadas e vítimas do comércio ilegal. Estas pesquisas podem ainda auxiliar gestores a identificar as principais espécies vítimas do tráfico e onde ocorrem com maior frequência, o que lhes permite subsidiar melhores políticas de fiscalização e conservação da fauna.

Lacunas de conhecimento nas áreas de pesquisa onde ainda há escassez de estudos também podem ser verificadas por meio da cienciometria. Neste estudo observouse que a maioria dos pesquisadores concentra suas pesquisas do tráfico de mamíferos. De fato, mamíferos são úteis para diversos fins à população em geral, o que lhes confere maior número de publicações. Entretanto há a necessidade de intensificar pesquisas com grupos taxonômicos menos abordados nos estudos, como invertebrados e a flora em geral.

Vale ressaltar ainda, que o tráfico de espécies é uma prática criminosa e que grande parte de informações sobre essa prática ilícita podem não ser obtidos nas pesquisas. Além disso, existe a necessidade de investimentos em pesquisas, 
principalmente em países em desenvolvimento, para estimular pesquisadores a ingressarem neste campo de pesquisa. Países que possuem rica biodiversidade são alvos de traficantes de espécies. O Brasil, por exemplo, pode oferecer um grande número de pesquisas sobre tráfico de espécies. No entanto é necessário mais investimento em educação, pesquisa e tecnologia para efetivar os estudos nesta área.

\section{Agradecimentos}

À agência de fomento Coordenação de Aperfeiçoamento de Pessoal de Nível Superior (CAPES) e à Pró-Reitoria de Pesquisa e Pós-Graduação da Universidade Estadual de Goiás pela concessão de bolsa de Pós-Graduação ao primeiro e ao segundo autor respectivamente.

\section{Referências}

Barbanera, F.et al.(2012). Conservation of endemic and threatened wildlife: Molecular forensic DNA against poaching of the Cypriot mouflon (Ovis orientalis ophion, Bovidae). Forensic Sci. Int.Genet., 6 (5), 671-675.

Barber-Meyer, S. M. (2010).Dealing with the clandestine nature of wildlife-trade market surveys. Conservation biology : the journal of the Society for Conservation Biology, 24 (4), 918-23.

Barbosa, F. G., Schneck, F., Melo, A. S. (2012).Use of ecological niche models to predict the distribution of invasive species: a scientometric analysis. Braz. J. Biol., 72 (4), 821-829.

Bastos, L. F., Luz, V. L. F., Reis, I. J., Souza, V. L. (2008). Apreensão de espécimes da Fauna Silvestre em Goiás Situação e destinação. Rev. Biol. Neotrop., 5(2), 51-63.

Brito, D., Oliveira, L. C., Oprea, M., Mello, M. A. R. (2009). An overview of Brazilian mammalogy: trends, biases and future directions. Zoologia, 26(1), 67-73.

Carneiro, F. M., Nabout, J. C., Bini, L. M. (2008). Trends in the scientific literature on phytoplankton. Limnology,9(2), 153-158.

Chaudhary, R. P. (2004).How to control illegal wildlife trade in the Himalayas - As Nepal's greatest natural resources approach extinction, the stakes could hardly be higher. Himalayan Journal of Sciences,2(3), 15-16.

Destro, G. F. G., Pimentel, T. L., Sabaini, R. M., Boorges, R. C., Barreto, R. (2012).Efforts to Combat Wild Animals Trafficking in Brazil. . In: G. A. Lameed (Ed.) Biodiversity Enrichment in a Diverse World (pp. 421-436). Croácia: INTECH.

Ferreira, C. M., Glock, L. (2004).Diagnóstico preliminar sobre a avifauna traficada no Rio Grande do Sul, Brasil. Biociências, 12 (1), 21-30.

Ferreira, S. M., Okita-Ouma, B. (2012).A proposed framework for short-, medium- and long-term responses by range and consumer States to curb poaching for African rhino horn. Pachyderm, 51, 52-59.

García, M. A. M., Suárez, C. (2000).El Tráfico Ilegal de Especies Silvestres. Cuardernos de Biodiversidad, 5, 12-14.

Glänzel, W., Leta, J., Thijs, B. (2006).Science in Brazil. Part 1: A macro-level comparative study. Scientometrics, 67 (1), 67-86.

Gong, S. P., Wang, J. C., Shi, H. T., Song, R. H., Xu, R. M. (2006).Illegal trade and conservation requirements of freshwater turtles in Nanmao, Hainan Province, China. Oryx, 40 (3), 331-336.

Greenacre, M., Hastie, T. (1987). The geometric interpretation of correspondence analysis. Journal of the American Statistical Association,82, 437-447.

Hansen, A. L. S., Li, A., Joly, D., Mekaru, S., Brownstein, J. S. (2012). Digital Surveillance: A Novel Approach to Monitoring the Illegal Wildlife Trade. Plos One, 7 (12), 112.
Hathaway, D. A. (2004). Biopirataria no Brasil. In: A. A. Rotania \& J. WERNECK (Eds) Sob o Signo das Bios - Vozes Críticas da Sociedade Civil (pp. 96). Rio de Janeiro: Epapers Serviços Editoriais.

Instituto Brasileiro de Geografia e Estatística - IBGE. (2004). Espécies extintas e ameaçadas de extinção. In: IBGE (Ed) Indicadores de desenvolvimento sustentável (pp. 100133). $1^{\text {a }}$ ed., Brasil.

Kuhl, A., et al. (2009). The role of saiga poaching in rural communities: Linkages between attitudes, socioeconomic circumstances and behaviour. Biological Conservation, 142(7), 1442-1449.

Lima-Ribeiro, M. D. S., et al. (2007). Análise cienciométrica em ecologia de populações: importância e tendências dos últimos 60 anos. Acta Scientiarum Biological Sciences, 29 (1), 39-47.

Macias-Chapula, C. A. (1998). 0 papel da informetria e da cienciometria e sua perspectiva nacional e internacional. Ci Inf., 27 (2), 134-140.

Maingi, J. K., Mukeka, J. M., Kyale, D. M., Muasya, R. M. (2012). Spatiotemporal patterns of elephant poaching in southeastern Kenya. Wildlife Research, 39 (3), 234-249.

Mayr R. M. (1998). The scientific investments of nations. Science,281 (5373), 49-51.

Nabout, J. C., et al.(2012). Trends and Biases in Global Climate Change Literature. Natureza \& Conservação, 10 (1), 45-51.

Peters, R. H. (1991). A critique for ecology. Cambridge: Cambridge University Press.

Pires, S. F., Clarke, R. V. (2011). Sequential foraging, itinerant fences and parrot poaching in Bolivia. Br. J. Criminol.,51 (2), 314-335.

Prado, L. A., Malheiros, R. (2012). A perda da biodiversidade do cerrado goiano mediante o tráfico de animais silvestres. Anais do III Congresso Brasileiro de Gestão Ambiental, 2 (1999), 1-12.

R Development Core Team R. (2011). A language and environment for statistical computing. R Foundation for Statistical Computing, Vienna, Austria.

Redford, K. H. (1992). The Empty Forest. BioScience, 42 (6), 412-422.

RENCTAS. 1을 Relatório Nacional sobre o Tráfico de Fauna Silvestre. 1aㅡ ed. Brasília, 2001.

RENCTAS. Rede Nacional de Combate ao Tráfico de Animais Silvestres. (2007). Vida silvestre: o estreito limiar entre preservação e destruição. Diagnóstico do Tráfico de Animais Silvestres na Mata Atlântica - Corredor Central e Serra do Mar. Brasília: Dupligráfica.

Ribeiro, L. B., Silva, M. G. (2002). 0 comércio ilegal põe em risco a diversidade das aves no Brasil. Ver. Ciência e Cultura, 59 (4), 4-5.

Saab, J. J. (2006). Tráfico ilícito de animais silvestres: a resposta penal segundo a lei 9.605/98. Revista Ciências Humanas, 12 (1), 61-66.

Schwartzman, S. A. (2003). Pesquisa Científica e o Interesse Público. Revista Brasileira de Inovação, 361-395.

Spinak, E. (1998). Indicadores cienciométricos. Ci. Inf.,27 (2), 141-148.

IUCN.(2013).The IUCN Red List of Threatened Species. Disponível em: <http://www. http://www.iucnredlist.org>Acesso em: 18/07/2013.

TRAFFIC. (2008).International Our Work: wildlife trade.2008. Disponível em:<http://www.traffic.org/trade>Acesso em: 18/07/2013. 